\title{
$\alpha 1$-antitrypsin promotes lung adenocarcinoma metastasis through upregulating fibronectin expression
}

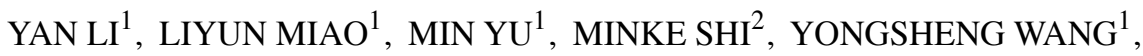 \\ $\mathrm{JUN}_{\mathrm{YANG}^{3}}{ }^{\text {, YONGLONG XIAO }}{ }^{1}$ and HOURONG CAI ${ }^{1}$ \\ Departments of ${ }^{1}$ Respiratory Medicine, ${ }^{2}$ Thoracic Surgery and ${ }^{3}$ Pathology, Nanjing Drum Tower Hospital, \\ The Affiliated Hospital of Nanjing University Medical School, Nanjing, Jiangsu 210008, P.R. China
}

Received November 14, 2016; Accepted March 29, 2017

DOI: $10.3892 /$ ijo.2017.3962

\begin{abstract}
AAT) has been recognized to be associated with lung adenocarcinoma metastasis. However, the mechanisms by which AAT promotes tumor metastasis remain to be investigated. Herein, we first examined AAT expression in a panel of formalin-fixed paraffin-embedded tumor tissues from 88 lung adenocarcinoma patients undergoing curative resection, using immunohistochemical methods. Lung adenocarcinoma patients with high AAT expression showed a significantly shorter overall survival compared to those with low AAT expression by Kaplan-Meier method $(\mathrm{P}=0.008)$. High AAT expression was also identified as an independent prognostic factor by Cox regression analysis (adjusted hazard ratio: $2.05 ; \mathrm{P}=0.04$ ). Second, the role of AAT in lung adenocarcinoma cell migration was evaluated in vitro using wound healing and Transwell assays, by transfecting the lentivirus vector with interfering sequence or coding sequence of AAT. The migration property of A549 and SPC-A1 cells was significantly diminished by downregulating AAT expression. Conversely, the migration of both cell lines was significantly increased through upregulating AAT. Furthermore, AAT could increase the expression of fibronectin (FN). FN downregulation reversed AAT-induced promotion of adenocarcinoma cell migration. Third, a cancer cell/endothelial cell co-culture model was established to investigate the effect of AAT on adenocarcinoma cell adhesion using immunofluorescence examination. The results showed that downregulation of AAT inhibited adhesion between lung adenocarcinoma cells and human umbilical vein endothelial cells whereas upregulation of AAT promoted adhesion, which may attribute to interactions between $\mathrm{FN}$ and integrin
\end{abstract}

Correspondence to: Dr Hourong Cai, Department of Respiratory Medicine, Nanjing Drum Tower Hospital, The Affiliated Hospital of Nanjing University Medical School, 321 Zhongshan Rd., Nanjing, Jiangsu 210008, P.R. China

E-mail: caihourong@yeah.net

Key words: al-antitrypsin, lung adenocarcinoma, fibronectin, metastasis, survival $\alpha 5$. Finally, AAT also showed the regulation effect on the metastatic behavior of lung adenocarcinoma cells in a mouse model, which may be through regulating FN expression. This study suggested that high AAT expression might be a negative prognostic marker for lung adenocarcinoma. AAT promoted lung adenocarcinoma metastasis, whose functional target may be FN. Our findings provide new insight into the mechanisms of lung adenocarcinoma metastasis.

\section{Introduction}

Adenocarcinoma has gradually become one of the most common pathological types of lung cancer. In clinical practice, most lung adenocarcinoma patients eventually suffered relapse and/or metastasis even when receiving complete excision of the cancer (1). Metastasis is the main cause of death in patients with lung adenocarcinoma (2). However, the underlying molecular mechanisms of cancer metastasis remain poorly understood.

a1-antitrypsin (AAT), also called serine proteinase inhibitor A1 (Serpin A1), is the most abundant serpin in human plasma. It is encoded by the protease inhibitor gene (SERPINA1) locus on the long arm of chromosome 14 (14q31-32.3). A major physiological role of AAT is to protect the lung from the destructive effects of excess uninhibited neutrophil elastase. It was shown that the serum levels of AAT were higher in cancer patients than in healthy controls $(3,4)$. Additionally, AAT has been found involving in the distant metastasis of lung adenocarcinoma (5). However, the mechanisms by which the increase of AAT promotes cancer metastasis remain undefined.

During the course of metastasis, cancer cells experience detachment, migration, invasion and adhesion. These key steps are inter-related and are affected by various biochemical factors. Fibronectin (FN) is one of the most abundant adhesion proteins and synthesized mainly by hepatocytes. Most FN circulates in the bloodstream as plasma $\mathrm{FN}$ while various cells also secrete FN, named cellular FN. Some malignant epithelial cells can produce $\mathrm{FN}$ and in some epithelial tumors FN was found upregulated $(6,7)$. FN plays an important role in cell growth, differentiation, migration and adhesion (8-10). FN could be recognized by various cell adhesion receptors, including integrins and dipeptidyl peptidase IV (DPP IV). 
Adhesion receptors in vascular endothelial cells can trigger an intracellular response when activated by ligands such as FN to facilitate cancer cell extravasating.

Integrins, which are members of a glycoprotein family, are the most well characterized receptors for FN. They are composed of $\alpha$ and $\beta$ subunits with non-covalent bonds connected to each other. There are at least 24 different integrin heterodimers that are dimerized by at least $19 \alpha$ and $8 \beta$ subunits and each integrin has distinct ligand binding and signaling properties (11). Endothelial cell surface express integrins which could recognize and are activated by ligands in the extracellular environment (12). Integrin $\alpha 5$ is encoded by the ITGA5 gene, which may mediate FN assembly (13). DPP IV is a $110-\mathrm{kDa}$ type II transmembrane sialoglycoprotein and its expression has been identified in various epithelial tissues including lung capillary endothelial cells (14-16). A major function of the DPP IV/FN adhesion has been reported in the colonization of the lungs by blood-borne cancer cells (17).

In this study, we investigated the prognostic effect of AAT expression on lung adenocarcinoma overall survival. Subsequently, we identified the effects of AAT on lung adenocarcinoma metastasis in vitro, specifically on cancer cell migration and the adhesion between cancer cells and vascular endothelial cells. The effects of FN in these processes were also explored by modulating its expression levels. Finally, we studied the influence of AAT and FN on metastatic colonization of lung adenocarcinoma cells in vivo.

\section{Materials and methods}

Clinical samples. Patients who underwent curative surgery and were diagnosed with lung adenocarcinoma in the department of thoracic surgery, Nanjing Drum Tower Hospital, the Affiliated Hospital of Nanjing University Medical School (Nanjing, China) between January 2003 and December 2009 were retrospectively reviewed. Eighty-eight patients with full medical records and follow-up information were identified and their formaldehyde fixed and paraffin embedded (FFPE) lung adenocarcinoma tumor tissues were collected. None of the 88 patients received radiation therapy or chemotherapy prior to surgery. The detailed information of the patients is shown in Table I. This study was approved by the Institutional Review Board and the Ethics Committee of Nanjing Drum Tower Hospital and written informed consent was obtained from each patient.

Immunohistochemistry. Adjacent 3- $\mu \mathrm{m}$ sections of FFPE samples were made for immunostaining. Each paraffin section was deparaffinized through dimethylbenzene and rehydrated by graded alcohols. After rehydration, antigen retrieval of the tisssue was carried by pressure cooking the slides with citric acid buffer ( $\mathrm{pH} 6.0$ for $1 \mathrm{~min}$ ). Rabbit polyclonal anti-human AAT antibody (1:100, Abcam, Cambridge, MA, USA) was used. AAT expression status was evaluated by two independent pathologists who were blinded to patient clinical characteristics (J. Yang and K. Meng). Five visual fields of each sample were randomly observed and 100 tumor cells in each field were counted (x400 magnification). Tumor cells with brown cytoplasm were considered positive and the staining intensity was rated as four classes: $3+$, strong; $2+$, moderate; $1+$, weak; and
0 , no staining. Positively stained cells out of 100 tumor cells in each field were recorded. The average percentage of positive tumor cells was categorized into the following four classes: 0 for $0 \%$; 1 for $1-33 \%$; 2 for $34-66 \%$; and 3 for $67-100 \%$. The scores of positive cell percentage and staining intensity were multiplied and composite scores of 1-3 were defined as low AAT expression, while scores of 4-9 were considered high AAT expression.

Cell lines. Human lung adenocarcinoma cell lines (A549 and SPC-A1) and human umbilical vein endothelial cell (HUVEC) were purchased from the Cell Bank of Chinese Academy of Medical Science (Shanghai, China) and maintained in our laboratory. A549 and SPC-A1 cells with stable green fluorescent protein (GFP) expression (A549/GFP and SPC-A1/GFP) were established in our laboratory through transfecting tumor cells with GFP expressing vector. All cell lines were cultured and maintained in recommended growth medium at $37^{\circ} \mathrm{C}$ in a $5 \% \mathrm{CO}_{2}$ humidified atmosphere.

Lentivirus vectors. Lentivirus vectors with interfering sequences (5'-AGTCCAACAGCACCAATAT-3' for AAT (pLenti-shRNA-AATi); 5'-TGGTTGTATCAGGACTTAT-3' for FN (pLenti-shRNA-FNi); 5'-ACTGTGGATCATCAT CCTA-3' for integrin $\alpha 5$ (pLenti-shRNA-ITGA5i); 5'-AGA AGACAACCTTGACCAT-3' for DPP IV (pLenti-shRNA-DDP IVi) or with coding sequence of AAT (pLenti-AAT) were purchased from Lifetech (Shanghai, China) and Genechem (Shanghai, China) companies. Lung adenocarcinoma cell lines were infected with a multiplicity of infection (MOI) as 1:10 in A549 cells and 1:100 in SPC-A1 cells. Polybrene (Sigma, St. Louis, MO, USA) at the concentration of $8 \mu \mathrm{g} / \mathrm{ml}$ was added to enhance the infection. Blasticidin $(0.5 \mu \mathrm{g} / \mathrm{ml})$ and puromycin $(1 \mu \mathrm{g} / \mathrm{ml})$ were used to screen the stable infected cells. The modulations of target gene expression were verified by real-time reverse transcription-PCR (RT-PCR).

Real-time RT-PCR. Total cellular RNA was extracted from A549 and SPC-A1 cells after transfecting with targeted gene interfering sequences (pLenti-shRNA-AATi or pLenti-shRNA-FNi) or coding sequence of AAT, respectively, using TRIzol (Invitrogen, Waltham, MA, USA). Subsequently, real-time RT-PCR was performed to determine AAT and FN expression in the StepOne System (Life Technologies, Carlsbad, CA, USA). RNA was also extracted from HUVEC after transfecting with targeted gene interfering sequences (pLenti-shRNA-ITGA5i or pLenti-shRNA-DDP IVi) and realtime RT-PCR was performed to determine integrin $\alpha 5$ and DPP IV expression, respectively. Relative gene expression was determined by the $\Delta \Delta \mathrm{Ct}$ method $(\Delta \Delta \mathrm{Cq})$ based on glyceraldehyde-3-phosphate dehydrogenase (GAPDH) levels (18), and results were expressed as fold change over different conditions.

Western blotting. After A549 and SPC-A1 cells were transfected with either pLenti-AAT or pLenti-shRNA-AATi, total cell protein were extracted from cells using RIPA buffer with proteinase inhibitors (Beyotime, Jiangsu, China) and then resolved by SDS-polyacrylamide gel electrophoresis and transferred to PVDF membranes. Membranes were blocked by $5 \%$ skim milk for 1 hour and incubated with rabbit poly- 
Table I. Association of AAT expression levels with clinicopathological characteristics in 88 resected lung adenocarcinoma patients.

\begin{tabular}{lccc}
\hline & $\begin{array}{c}\text { High AAT } \\
\text { expression } \\
\text { no. }(\%)\end{array}$ & $\begin{array}{c}\text { Low AAT } \\
\text { expression } \\
\text { no. } \%)\end{array}$ & P-value \\
\hline Age (years) & & & 0.48 \\
$\quad$ 60 & $25(45.5)$ & $16(48.5)$ & \\
$>60$ & $30(54.5)$ & $17(51.5)$ & \\
Sex & & & 0.27 \\
Female & $25(45.5)$ & $18(54.5)$ & \\
Male & $30(54.5)$ & $15(45.5)$ & \\
Smoking status & & & 0.22 \\
Never smokers & $36(65.5)$ & $25(75.8)$ & \\
Smokers & $19(34.5)$ & $8(24.2)$ & \\
Tumor differentiation & & & 0.24 \\
Well+Moderately & $33(60.0)$ & $23(69.7)$ & \\
Poorly & $22(40.0)$ & $10(30.3)$ & \\
Regional lymph node metastasis & & & \\
No & $24(43.6)$ & $22(66.7)$ & $0.03^{\mathrm{a}}$ \\
Yes & $31(56.4)$ & $11(33.3)$ & \\
pTNM stage & & & 0.07 \\
I & & & \\
II & $15(27.3)$ & $17(51.5)$ & \\
III & $21(38.2)$ & $9(27.3)$ & \\
\hline
\end{tabular}

AAT, $\alpha 1$-antitrypsin; ${ }^{\text {a }}<0.05$ was considered to be significant.

clonal anti-human AAT antibody (1:100, Abcam) overnight at $4^{\circ} \mathrm{C}$, followed by incubation with appropriate HRP-conjugated secondary antibody at optimized concentration. The densitometry of western blot results was measured using ImageJ software (https://imagej.nih.gov/ij/).

Immunofluorescence studies. In order to demonstrate the influence of AAT on FN expression and observe the adhesion between adenocarcinoma cells and HUVECs under various conditions, immunofluorescence was applied to detect the AAT and FN expression levels in tumor cells and CD31 in HUVECs, with 4',6-diamidino-2-phenylindole (DAPI) for nuclear staining. Rabbit anti-human FN antibody and rabbit monoclonal anti-human CD31 antibody (1:200, Abcam) were used. Rabbit polyclonal anti-human FN antibody (1:100, Abcam) was applied with a FITC conjugated rat anti-rabbit secondary antibody and rabbit monoclonal anti-human CD31 antibody (1:200, Abcam) was applied to identify the HUVECs with a Cy3 conjugated rat anti-rabbit second antibody.

Wound healing and Transwell assay. The migration ability of adenocarcinoma cells was determined by wound healing assay and Transwell assay. AAT expression levels in lung adenocarcinoma cells were modulated by pLenti-AAT or pLenti-shRNA-AATi. Human lung adenocarcinoma cells (A549 and SPC-A1) were cultured in 6-well culture plate
( $2.5 \times 10^{5}$ per well) for $24 \mathrm{~h}$ until $90 \%$ confluent. Wound line was created by scratching the plates with a $20 \mu 1$ micropipette tip. Migration rate of tumor cells was calculated by the following formula: healing rate $=$ (the distance before healingthe distance after healing)/the distance before healing $\mathrm{x} 100 \%$, in which the distances of gaps were recorded at identical point.

Transwell chambers $(8 \mu \mathrm{m}$ pore size, Costar, Fisher Scientific, MA, USA) were also applied. Cells suspended in $100 \mu \mathrm{l}$ of medium $\left(1 \times 10^{5}\right.$ cells $)$ were placed into the top chamber and $600 \mu \mathrm{l}$ conditioned medium was added to the bottom well. Cells adherent to the upper surface of the membrane were removed using a cotton applicator after $24 \mathrm{~h}$ incubation. After fixation in ice cold acetone, cells on the bottom surface of membrane were stained with $0.4 \%$ violet crystal, and counted by microscopy.

Cell adhesion examination. To analyze the effect of AAT on adhesion of lung adenocarcinoma cells to vascular endothelial cells, a cancer cell/endothelial cell co-culture model was established. First, HUVECs were seeded on sterile cover glasses and cultured in 6-well plates with endothelial cell growth medium and growth supplement (Sigma). When HUVECs on cover glasses grew to confluent, all the cover glasses were transferred into new 6-well plates. At the same time, single cell solutions of A549/GFP and SPC-A1/GFP cells were collected and seeded into 6-well plates in which cover glasses with confluent HUVECs had been placed. After $2 \mathrm{~h}$ co-culture, the cover glasses were collected and washed with PBS, followed by fixation in cold acetone for immunofluorescence examination.

Expression levels of AAT and FN in tumor cells and expression levels of integrin $\alpha 5$ and DPP IV in HUVECs were modulated by lentiviral-mediated deliveries of coding sequence or interfering sequence.

Animal models. T cell deficient BALB/c nude mice (CByJ. Cg-Foxn $\left.1^{n u} / \mathrm{J}\right)$ were purchased from Model Animal Research Center of Nanjing University. Animal use and experiment protocol were approved by the Institutional Animal Care and Use Committee of Nanjing Drum Tower Hospital. All surgery was performed under anesthesia with pentobarbital sodium and ketamine. Animals were sacrificed by overdose of anesthetics.

Cancer cells (A549 and SPC-A1, respectively) with AAT upregulation or AAT downregulation were cultured and injected through lateral tail vein of nude mice (female, 4-6 weeks old) with a total cell number of $1 \times 10^{6}$ per mouse. Mice were divided into following six groups: 1) normal control (NC) in A549; 2) AAT upregulation alone in A549; 3) AAT upregulation followed by FN downregulation in A549; 4) NC in SPC-A1; 5) AAT upregulation alone in SPC-A1; 6) AAT upregulation followed by FN downregulation in SPC-A1. A total of six mice in each group were used. Two months after injection, total lung tissues of mice were collected and embedded in paraffin. The whole lung tissues were analyzed by consecutive sections and hematoxylin and eosin (H\&E) staining. Lung metastasis loci were counted by microscopy.

Statistical analysis. Differences between clinicopathological variables and AAT expression levels were examined by Chi-square test or Fisher's exact test if any sample number $<5$. 

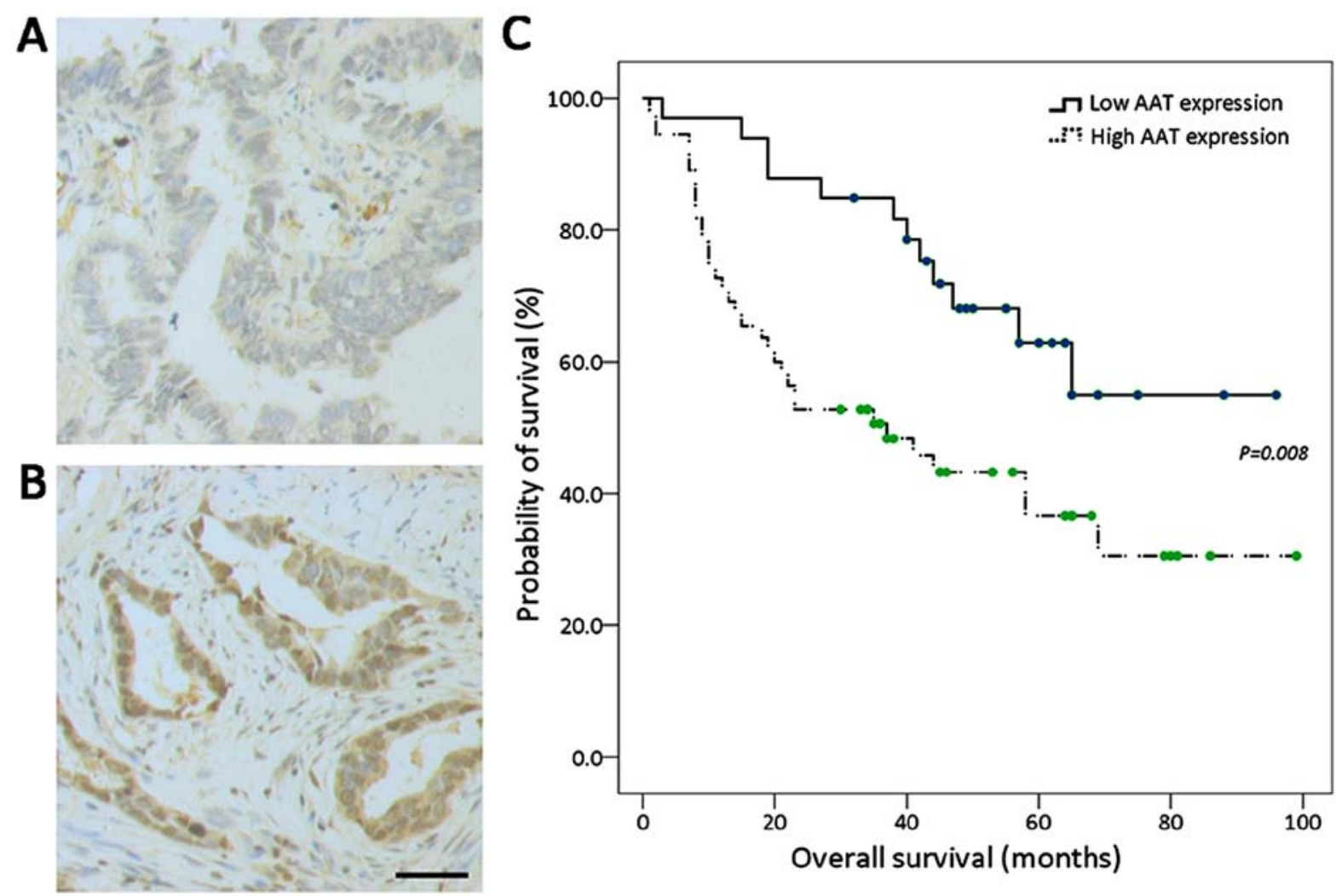

Figure 1. Clinicopathological significance of $\alpha 1$-antitrypsin (AAT) expression in lung adenocarcinoma. According to the score of immunohistochemistry in lung adenocarcinoma tumor tissue, all the cases were classified as AAT low expression (A) or high expression (B). AAT was mostly expressed in the cytoplasm of lung adenocarcinoma cells. (C) Lung adenocarcinoma patients with high AAT expression in tumor samples had a shorter overall survival time ( $\mathrm{P}=0.008)$. Scale bar, $100 \mu \mathrm{m}$.

Overall survival (OS) was calculated as the time from the date of lung surgery to death. Patients who were alive at the last contact were censored. Kaplan-Meier method and log-rank test were used to calculate the survival difference. Univariate Cox regression analysis was used to evaluate the prognostic impact on OS of clinicopathological variables. Variables were included in multivariate analysis at $\mathrm{P}<0.05$ in the univariate analysis. Multivariate Cox regression analysis was used to evaluate the independent prognostic role of AAT expression. The experimental results are shown as mean \pm SEM. One-way ANOVA, LSD and unpaired t-test were used to analyze the differences between groups. All analyses were performed with SPSS software, version 16.0 (SPSS, Inc., Chicago, IL, USA). All tests were two-sided and performed at a significance level of 0.05 .

\section{Results}

High expression of AAT in tumor tissue is related to shorter overall survival of lung adenocarcinoma patients. Positive immunohistochemistry staining for AAT was mainly localized in the cytoplasm. According to the score of immunohistochemistry, all lung adenocarcinoma cases were classified as low AAT expression group (33/88, Fig. 1A) and high expression group (55/88, Fig. 1B). Clinicopathological characteristics of included lung adenocarcinoma patients are listed in Table I by AAT expression status. There were no significant differences in age, gender, smoking status, tumor differentiation, or pTNM stage between patients with high AAT expression and those with low AAT expression $(\mathrm{P} \geq 0.05)$. The correlation between regional lymph node metastasis and AAT expression was also examined, with more cases showing regional lymph node metastasis in the high AAT expression group (56.4\% vs. $33.3 \%, \mathrm{P}=0.03$ ). The median OS of 88 resected lung adenocarcinoma patients was 58.0 months (95\% CI: 37.7-78.3). As indicated in Fig. 1C, lung adenocarcinoma patients with high AAT expression in tumor samples had shorter OS than those with low AAT expression $(\mathrm{P}=0.008)$. In univariate $\mathrm{Cox}$ regression analysis, as shown in Table II, tumor differentiation, regional lymph node metastasis and pTNM stage associated significantly with OS $(\mathrm{P}<0.05)$. There was a significant association of AAT high expression level with shorter OS (hazard ratio: $2.38 ; 95 \%$ CI: 1.23, 4.63; $\mathrm{P}=0.01$ ). In multivariate analysis adjusting for variables significant in the univariate analysis, the significant association of AAT expression with OS remained (adjusted hazard ratio: 2.05; 95\% CI: 1.04-4.06; $\mathrm{P}=0.04$ ).

AAT promotes the migration ability of lung adenocarcinoma cells in vitro by regulating $F N$ expression. AAT expression level was significantly higher after transfecting with the AAT coding sequence pLenti-AAT; while it was significantly lower after transfecting with the AAT interfering sequence pLentishRNA-AATi, in both A549 cell line and SPC-A1 cell line (Fig. 2A and B). The effect of AAT on FN expression was investigated by immunofluorescence, which showed that FN expression increased upon upregulation of AAT expression 


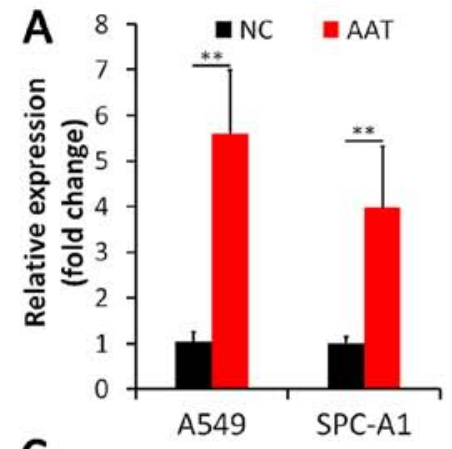

C
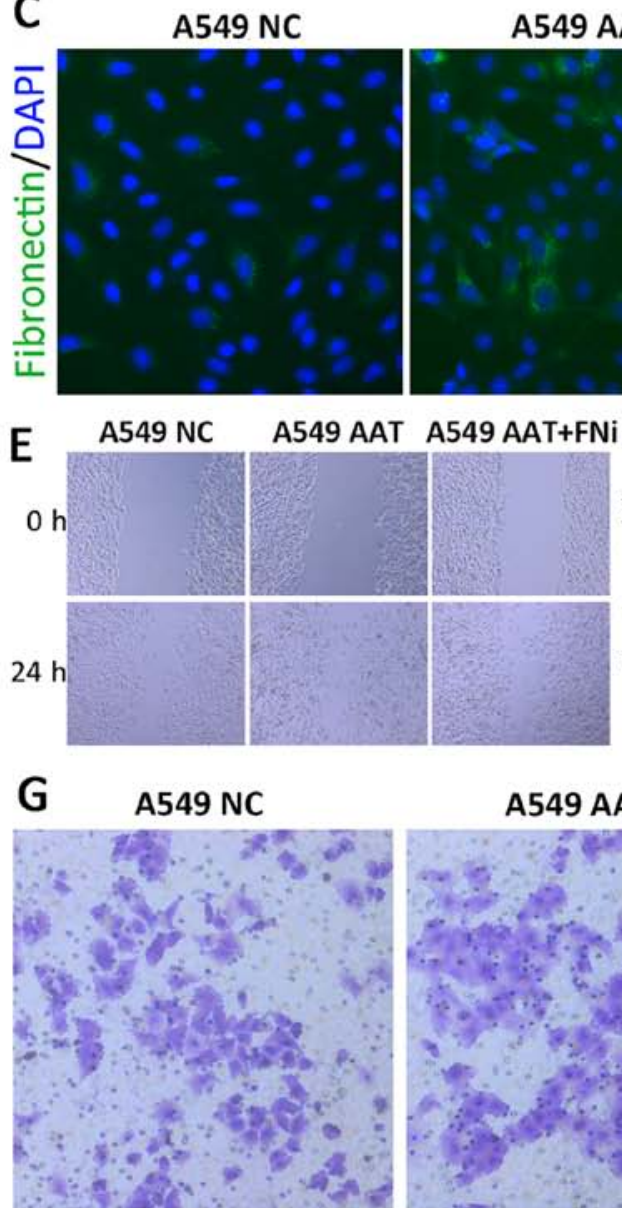

H
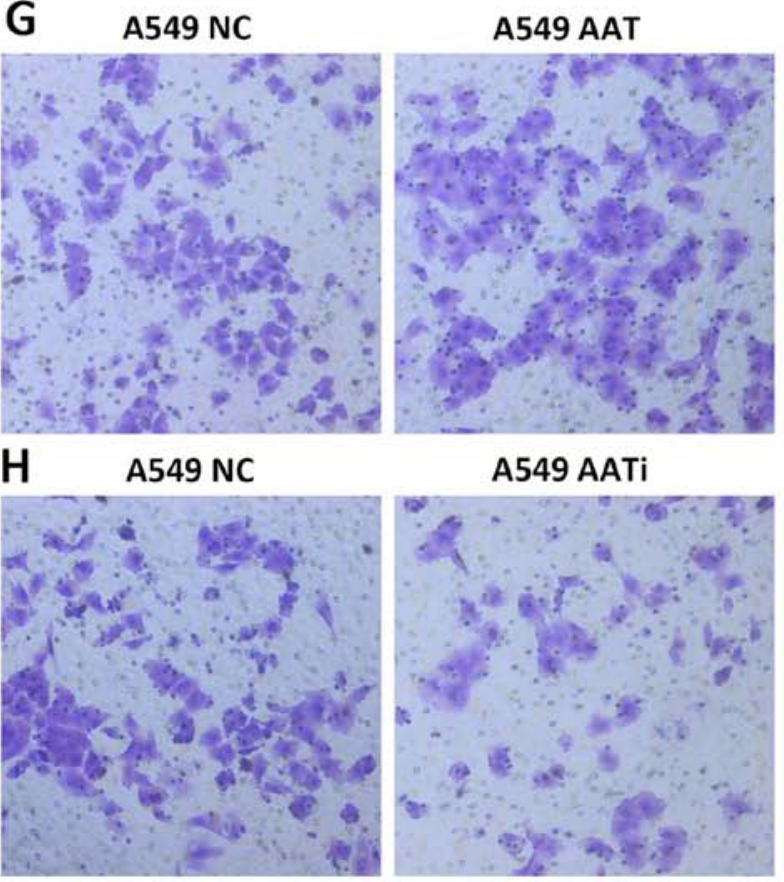
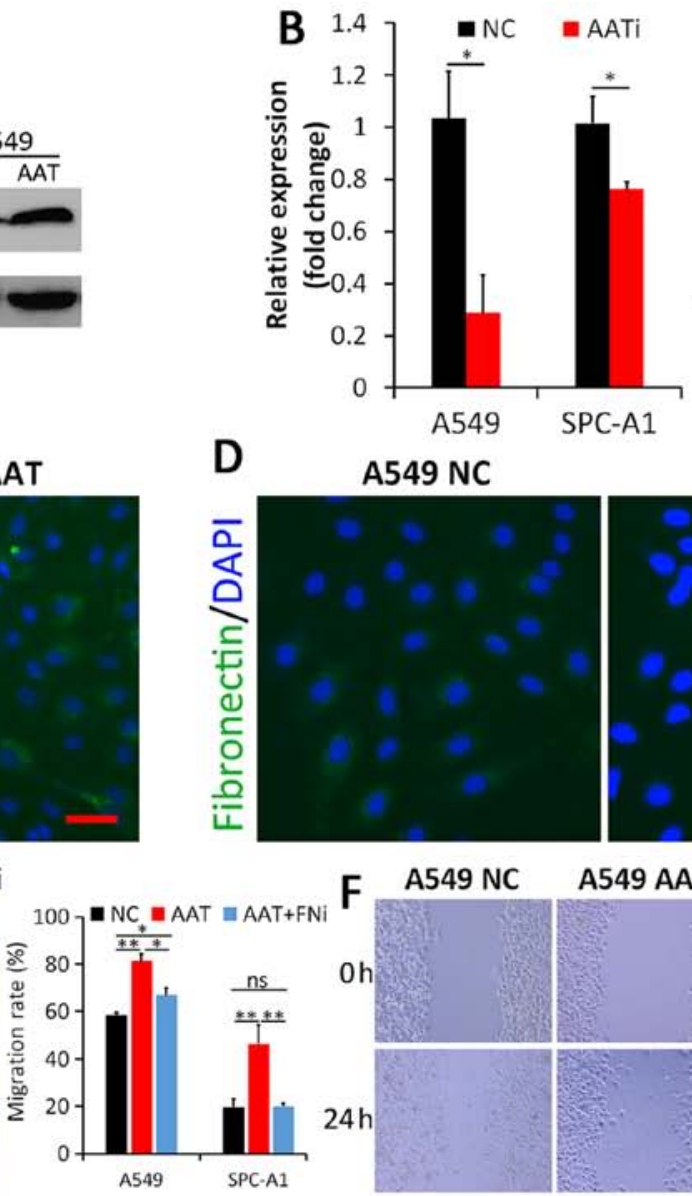

D

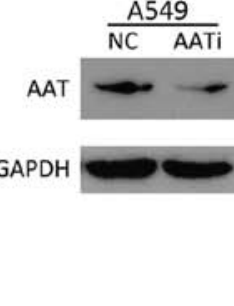

A549 AATi

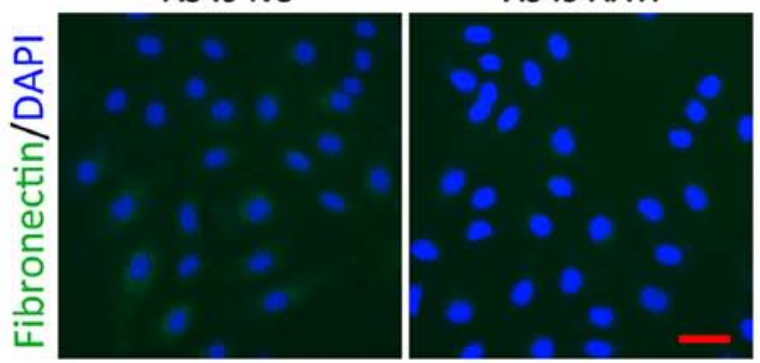

\section{F $A 549 \mathrm{NC} \quad$ A549 AATi}
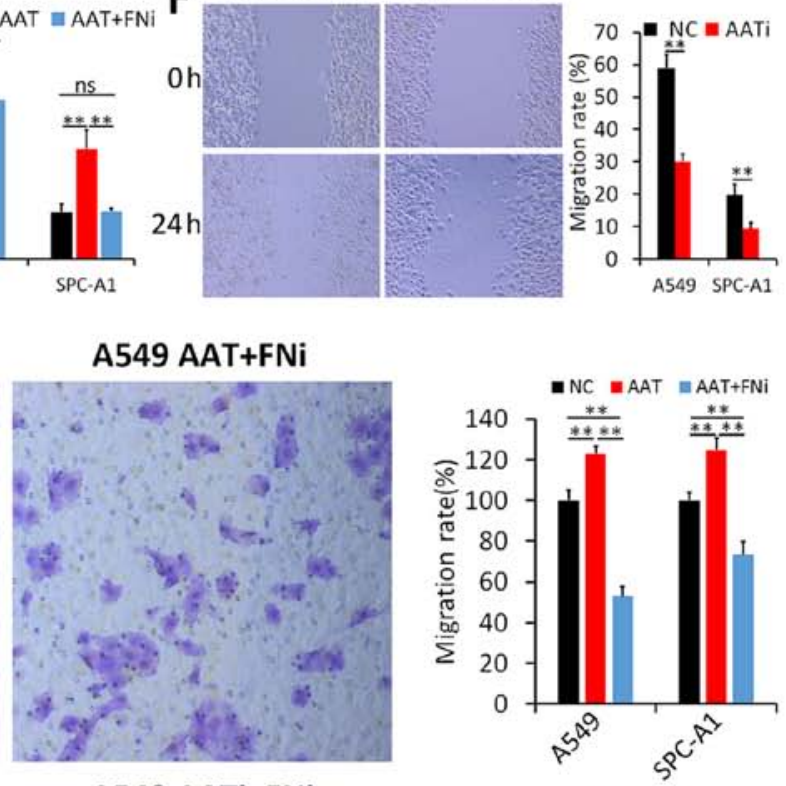

A549 AATi+FNi
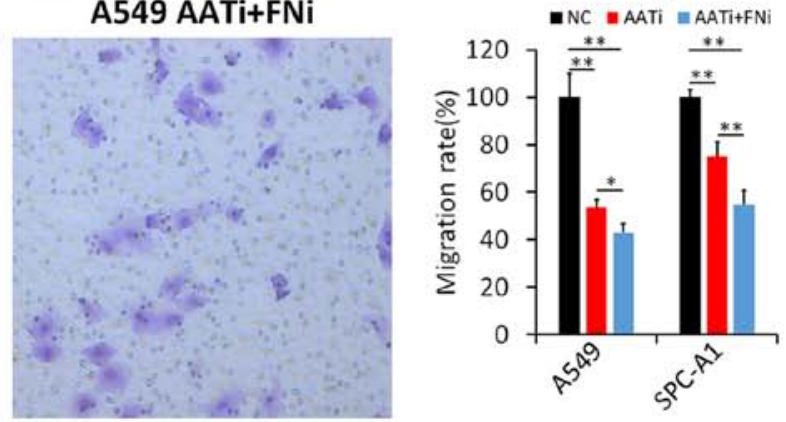

Figure 2. $\alpha$ 1-antitrypsin (AAT) regulates the expression of fibronectin $(\mathrm{FN})$ in lung adenocarcinoma cells and promotes migration of lung adenocarcinoma cells. Overexpression and knockdown of AAT mediated by lentivirus vectors were verified by RT-PCR and western blotting (A and B). GAPDH was used as internal control. (C and D) Representative immunofluorescence staining of FN (green) in A549 cells with 4',6-diamidino-2-phenylindole (DAPI) for nuclear staining (blue). FN was expressed in cytoplasm and surface of lung adenocarcinoma cells. Upregulation of AAT could increase the expression of FN and vice versa. (E and F) Cell wound healing assay. The extent of closure was photographed at 0 and $24 \mathrm{~h}$ after treatment at $\mathrm{x} 100 \mathrm{magnification}$. AAT prompts migration of A549 cells and SPC-A1 cells (E). When AAT was knocked down, the migration rate of A549 cells and SPC-A1 cells were reduced (F). Downregulating the expression of FN could inhibit the migration of lung adenocarcinoma cells even though AAT was upregulated (E). (G and $\mathrm{H}$ ) Transwell migration assay at $\mathrm{x} 200$ magnification. Migration was quantified by counting cells in six random fields per membrane. In accordance with data in E and F, AAT affects the migration of lung adenocarcinoma cells and FN interfering could reverse the promotion effect induced by AAT high expression. Each assay was performed in independent biological triplicates. The data are presented as the mean \pm SD. Bars indicate standard deviation. AAT, AAT overexpression; AATi, AAT knockdown; FNi, FN knockdown. ${ }^{*} \mathrm{P}<0.05,{ }^{* *} \mathrm{P}<0.01$. Scale bar in $\mathrm{C}$ and $\mathrm{D}, 100 \mu \mathrm{m}$. 

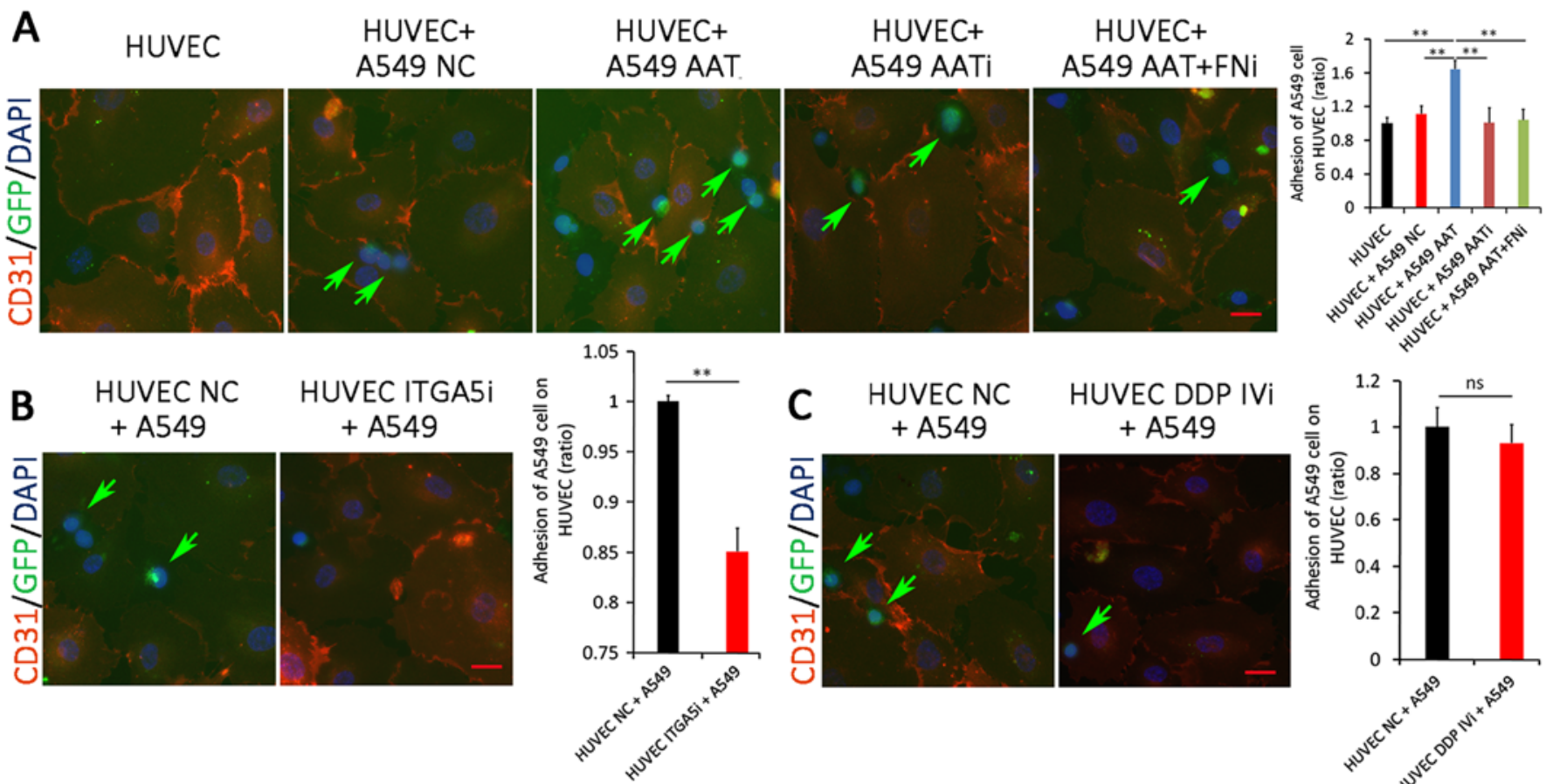

D
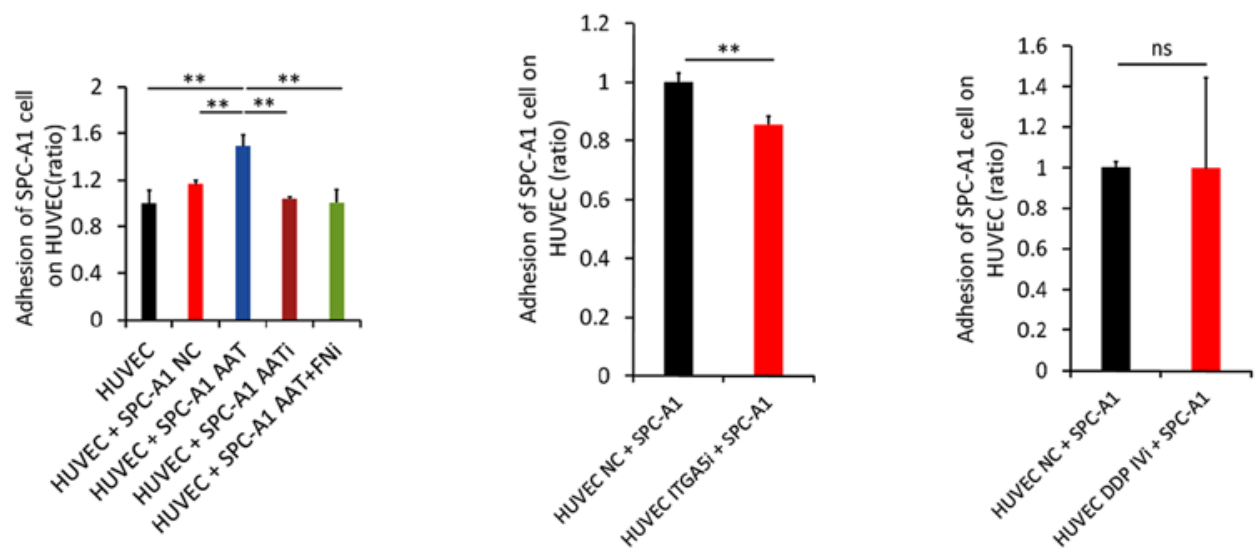

Figure 3. $\alpha 1$-antitrypsin (AAT) enhances adhesion between lung adenocarcinoma cells and endothelial cells. Human umbilical vein endothelial cells (HUVECs) were stained with aniti-CD31 antibody (red). A549 cells stained green were considered as expressing GFP and all nuclei were stained by DAPI (blue). (A) Upregulation of AAT enhances the adhesion ability of A549 cells and more GFP positive A549 cells adhered to HUVECs. Downregulation of AAT decreased the adhesion ability. Fibronectin (FN) downregulation reversed the enhanced adhesion induced by AAT upregulation. (B) Downregulation of integrin $\alpha 5$ of HUVECs inhibited adhesion between GFP positive A549 cells and HUVECs. (C) The adhesion between A549 cells and HUVECs was not affected when DPP IV was knocked down in HUVECs. (A-C, left panel, representative images; right panel, quantitative data). (D) Similar results were demonstrated in SPC-A1 cells. Red arrows, GFP positive A549 cells. Scale bar, $100 \mu \mathrm{m}$. ${ }^{*} \mathrm{P}<0.05,{ }^{* *} \mathrm{P}<0.01$. Scale bar, $100 \mu \mathrm{m}$.

while decreased upon downregulation of AAT expression in both A549 cell line (Fig. 2C and D) and SPC-A1 cell line (data was not shown). These findings demonstrated that regulating AAT expression could affect FN expression in lung adenocarcinoma cells.

Wound healing assay and Transwell assay then were used to examine the effects of AAT and FN on adenocarcinoma cell migration ability. First, A549 and SPC-A1 cells were transfected with pLenti-AAT or control vectors respectively. The results of the wound healing assay showed that the migration rate of adenocarcinoma cells with AAT upregulation was significantly higher than that of parental cells transfected with control vectors $(\mathrm{P}<0.01$, Fig. $2 \mathrm{E})$. Similarly, AAT downregulation impeded wound healing rate of adenocarcinoma cells $(\mathrm{P}<0.01$, Fig. 2F). Second, to examine whether adenocarcinoma cells with higher AAT expression contribute their increased migration ability to FN, FN expression was inhibited by transfecting Lenti-shRNA-FNi in lung adenocarcinoma cells with AAT upregulation. We found that FN downregulation could reverse the increased migration induced by AAT upregulation in adenocarcinoma cells $(\mathrm{P}<0.05$, Fig. 2E). Furthermore, Transwell assay was performed. As shown in Fig. $2 \mathrm{G}$ and Fig. $2 \mathrm{H}$, the transmigration ability of A549 were significantly increased when transfected with pLenti-AAT, compared to the control group $(\mathrm{P}<0.01)$. Conversely, the cells showed decreased transmigration upon trasfection of pLenti-AATi. In addition, when FN expression was inhibited, the transmigration rate significantly decreased even though AAT was upregulated, compared to those without FN interference $(\mathrm{P}<0.01)$. If both FN and AAT expression levels were inhibited, A549 cells 

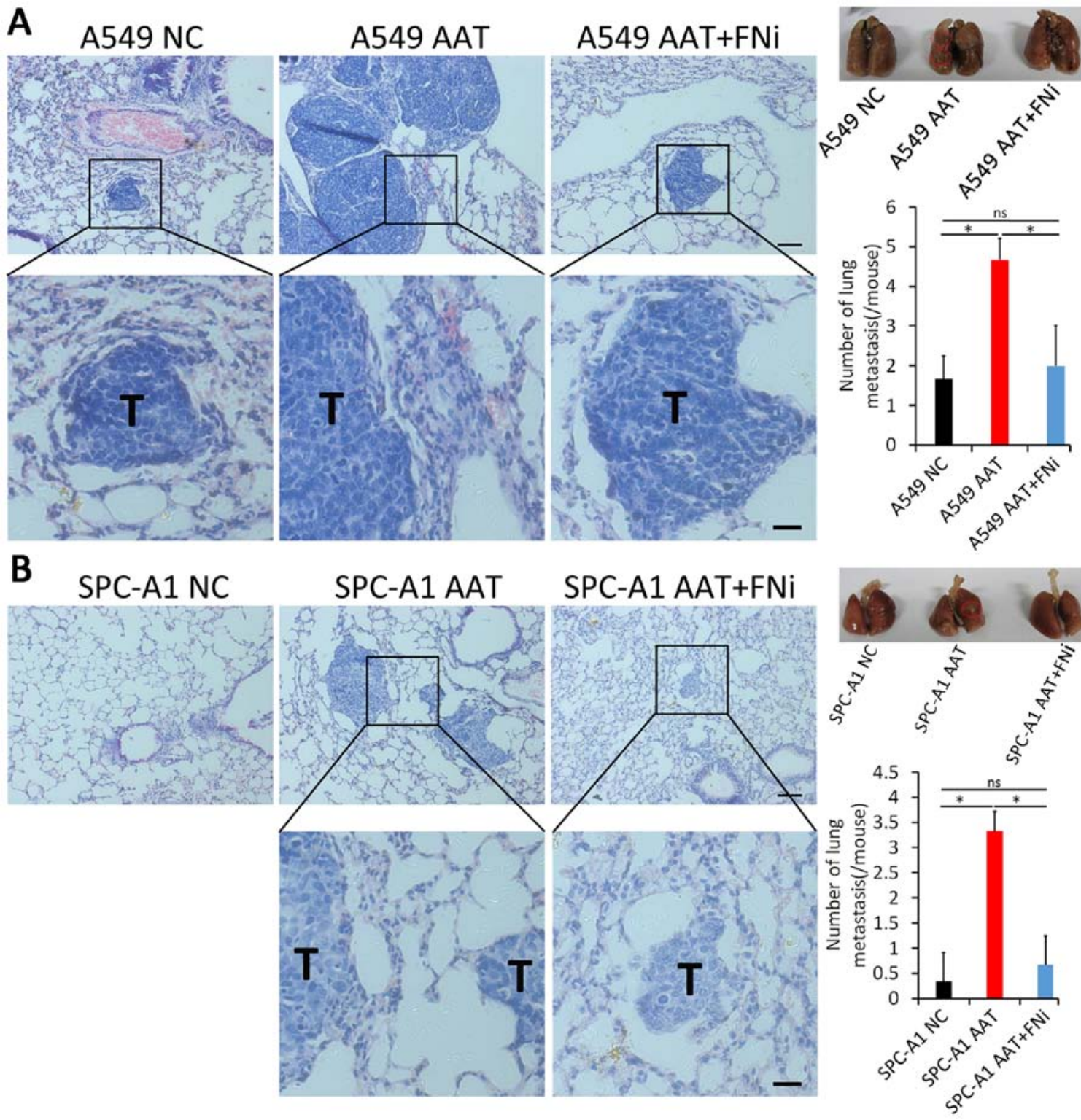

Figure 4. $\alpha 1$-antitrypsin (AAT) promotes hematogenous metastasis of lung adenocarcinoma cells. Hematogenous metastasis models of lung adenocarcinoma cells were established by injecting tumor $\left(1 \times 10^{6}\right.$ cells per mouse) into nude mice through the tail vein. Lung metastasis nodules were examined by hematoxylin and eosin (H\&E) staining of the lung tissues. (A) Overexpression of AAT prompts metastasis of A549 cells (left panel, representative images; right upper panel, metastasis nodules in lung tissues indicated by red circles; right lower panel, quantitative data). Downregulating the expression of fibronectin (FN) could inhibit the metastasis of A549 cells even though AAT was upregulated. (B) In accordance with data in A, AAT affects the metastasis of SPC-A1 cells and FN interfering could reverse the promotion effect induced by AAT high expression. AAT, AAT overexpression; FNi, FN knockdown. Black T, lung metastasis nodules. ${ }^{*} \mathrm{P}<0.05$, Scale bar, $100 \mu \mathrm{m}$.

showed further decreased transmigration compared to those with only AAT inhibited $(\mathrm{P}<0.05)$. Similar results were also observed in SPC-A1 cells $(\mathrm{P}<0.01)$. These findings indicate that the effect of AAT on lung adenocarcinoma cell migration might be related to the expression of $\mathrm{FN}$.

AAT promotes adhesion between lung adenocarcinoma cells and vascular endothelial cells through regulating FN expression. To gain further insight into the underlying molecular mechanism by which AAT promotes lung adenocarcinoma metastasis, a cancer cell/endothelial cell co-culture model was established. As indicated in Fig. 3A, more GFP-positive A549 cells adhered to HUVECs when AAT was upregulated $(\mathrm{P}<0.01)$. However, downregulation of AAT did not further inhibit adhesion between A549 cells and HUVECs compared to those with the control vector. Additionally, the adhesion of A549 cells to HUVECs significantly decreased if their FN expression was impaired, compared to those with AAT upregulation alone $(\mathrm{P}<0.01)$. These results suggest that the promotion effect of AAT on the adhesion ability of lung adenocarcinoma cells to endothelial cells might be through the effect of FN. Similar results were also observed in SPC-A1 cells (Fig. 3D).

The functions of FN receptors on vascular endothelial cells were further examined by RNA-interference-based studies. Two reported receptors of FN, integrin $\alpha 5$ and DPP IV, were inhibited in HUVEC, respectively. As indicated in Fig. 3B, downregulation of integrin $\alpha 5$ inhibited adhesion between 
Table II. Prognostic value of clinicopathological characteristics and AAT expression in univariate Cox regression analysis.

\begin{tabular}{|c|c|c|}
\hline Patient characteristics & $\begin{array}{l}\text { Unadjusted HR } \\
(95 \% \mathrm{CI})\end{array}$ & P-value \\
\hline \multicolumn{3}{|l|}{ Age (years) } \\
\hline$\leq 60$ & Reference & \\
\hline$>60$ & $1.07(0.60-1.93)$ & 0.81 \\
\hline \multicolumn{3}{|l|}{ Gender } \\
\hline Female & Reference & \\
\hline Male & $1.51(0.83,2.75)$ & 0.17 \\
\hline \multicolumn{3}{|l|}{ Smoking status } \\
\hline Never smokers & Reference & \\
\hline Smokers & $1.17(0.63,2.18)$ & 0.62 \\
\hline \multicolumn{3}{|l|}{ Tumor differentiation } \\
\hline Well+Moderately & Reference & \\
\hline Poorly & $2.03(1.13,3.64)$ & $0.02^{\mathrm{a}}$ \\
\hline \multicolumn{3}{|c|}{ Regional lymph node metastasis } \\
\hline No & Reference & \\
\hline Yes & $2.33(1.27,4.27)$ & $0.006^{\mathrm{a}}$ \\
\hline \multicolumn{3}{|l|}{ pTNM stage } \\
\hline I & Reference & \\
\hline II & $2.40(1.09,5.25)$ & $0.03^{\mathrm{a}}$ \\
\hline III & $3.36(1.54,7.31)$ & $0.002^{\mathrm{a}}$ \\
\hline \multicolumn{3}{|l|}{ AAT expression level } \\
\hline Low & Reference & \\
\hline High & $2.38(1.23,4.63)$ & $0.01^{\mathrm{a}}$ \\
\hline
\end{tabular}

AAT, $\alpha 1$-antitrypsin; ${ }^{\text {a }}<0.05$ was considered to be significant.

GFP-positive A549 cells and HUVECs $(\mathrm{P}<0.01)$. However, when DPP IV was knocked down in HUVECs, the adhesion between A549 cells and HUVECs were not significantly inhibited (P>0.05, Fig. 3C). Similar results were also observed in SPC-A1 cells (Fig. 3D). Collectively, these results suggest that FN may be a functional target of AAT, which is responsible for AAT-mediated adhesion between lung adenocarcinoma cells and endothelial cells.

AAT promotes metastasis of lung adenocarcinoma in vivo. To further confirm whether AAT could promote the metastatic behavior in vivo, A549 cells with AAT overexpression were injected into nude mice through the tail vein. As shown in Fig. 4A, the number of lung metastatic loci in the AAT overexpression group was remarkably higher compared to the NC group $(\mathrm{P}<0.05)$. We further examined the effect of FN on lung colonization of A549 cells by downregulating FN expression in addition to upregulating AAT expression in immunocompromised nude mice. Results showed that downregulation of FN could induce decreased lung metastasis even though AAT was upregulated (Fig. 4A, $\mathrm{P}<0.05$ ). Similar results were observed in SPC-A1 cells (Fig. 4B, P<0.05). The results indicate that AAT could significantly promote the metastasis

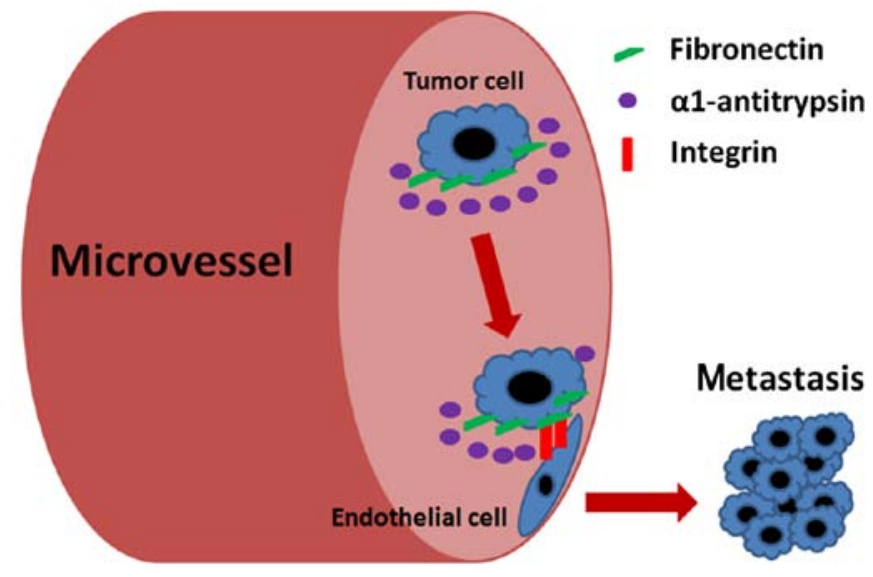

Figure 5. The mechanism by which $\alpha 1$-antitrypsin promotes metastasis of lung adenocarcinoma. Lung adenocarcinoma cells might increase the concentration of $\alpha 1$-antitrypsin around tumor cells, and thereafter decrease the degradation of fibronectin on tumor cells. $\alpha 1$-antitrypsin was able to promote metastasis of lung adenocarcinoma cells mediated by the combination of fibronectin (on tumor cells) and integrin (on microvessel endothelial cell) which might be the key procedure for extravasation of tumor cells.

of lung adenocarcinoma cells in the nude mouse xenograft model, which may be through regulating FN expression.

\section{Discussion}

In this study, we investigated the effect of $\alpha 1$-antitrypsin (AAT) on lung cancer metastasis. We found that AAT expression was associated with overall survival in resected lung adenocarcinoma. Furthermore, we identified that AAT was able to promote migration and adhesion of lung adenocarcinoma cells through enhancing the expression of FN. In the experiment with nude mouse xenograft model, AAT upregulation could significantly promote metastasis of lung adenocarcinoma cells, while FN downregulation could reverse the promotion effect. These results suggested that AAT may be a therapeutic target for lung adenocarcinoma metastasis.

Metastasis is the most critical complication of malignancies and remains a big challenge to the effective treatment for lung adenocarcinoma. Our understanding of the molecular mechanisms regarding lung adenocarcinoma metastasis is still incomplete. AAT is a glycoprotein synthesized primarily by hepatocytes, with smaller amounts synthesized by intestinal epithelial cells, neutrophils, pulmonary alveolar cells, macrophages, and cancer cells $(19,20)$. AAT has long been recognized as an important anti-protease which protects the lung from the destructive effects of major proteases such as neutrophil elastase. In recent years, AAT has gradually been found to have an impact on lung cancer metastasis. Increased serum AAT concentration was a poor prognostic marker for non-small cell lung cancer (21). In this study, we demonstrated that AAT expression in tumor tissue was a prognostic marker for lung adenocarcinoma patients, with higher expression associated with shorter overall survival. Our immunohistochemistry results showed a positive correlation between AAT expression and the frequency of regional lymph node involvement, suggesting that AAT overexpression could be important for the remodeling process during lung adenocarcinoma development and metastasis. However, the molecular mechanism of 
AAT influencing cancer metastasis has still not been completely defined.

The migratory ability of cancer cells is one critical parameter of the metastatic cascade. Our findings demonstrated that AAT overexpression could promote migration of lung adenocarcinoma cells, but this promotion effect could be reversed by FN downregulation. FN plays key roles in promoting oncogenic transformation and has been associated with cell migration and invasion in various malignancies, including lung cancer $(22,23)$. Digiacomo et al found that FN stimulated the migration of murine or human macrophages and the activation of SFK/FAK complex, while the macrophage migration depended on FAK activity (24). This phenomenon may be extended to explain the effect of FN on tumor cell migration. FN could interact with the integrins and then lead to the activation of many signaling pathways, including c-Met/FAK/Src and FAK-PI3K/Akt pathways, which regulate cancer cell adhesion and migration $(25,26)$. Mitra et al suggested that FN may bind integrin $\alpha 5 \beta 3$ on cancer cells and subsequently activate the FAK/ Src-dependent signaling pathway (27). FN could stimulate the secretion of MMP-9 through the MEK1/ERK and the PI-3K/Akt-dependent pathways in breast cancer cells, thereby triggering invasion of tumor cells (28). Thus, FN may be a significant factor in the process of AAT promoting migration of lung adenocarcinoma cells.

We have examined the mRNA levels of FN in lung adenocarcinoma cells (A549 and SPC-A1) after upregulation or downregulation of the AAT expression. There was no significant correlation between mRNA levels of FN and AAT in the two cell lines. As a result, AAT might regulate the expression of FN through an indirect way. As indicated in Fig. 2, FN protein was upregulated in both cytoplasm and surface of lung adenocarcinoma cells after upregulation of AAT. We hypothesized that AAT could prevent degradation of FN intraand extra-adenocarcinoma cells. It has been widely accepted that the physiological role of AAT is to inhibit the destructive effects of excess uninhibited neutrophil elastase (19), which means that AAT could prevent degradation of FN through inhibiting proteases.

Once tumor cells are circulating, they are in a suspended state and require additional cellular activities to enable their colonization in distant organs, which is mostly initiated by adhesive interactions with the endothelium. In the present study we showed that the adhesion between lung adenocarcinoma cells and vascular endothelium was regulated by expression levels of AAT and FN. FN could adhere to integrin $\alpha 5$ expressed on the endothelium. The specificity of integrin $\alpha 5 / \mathrm{FN}$ adhesion was confirmed by reduced adhering ability when lung adenocarcinoma cells were treated with integrin $\alpha 5$ interfering sequence. Interactions between $\mathrm{FN}$ and integrins play important roles in cancer metastasis (29). Through specifically binding to integrins, such as $\alpha 5 \beta 1$ or $\alpha 5 \beta 3$, FN may activate multiple signal pathways, and then regulate malignant cellular metastasis $(30,31)$.

In our study, however, DDP IV failed to show significant effect on adhesion between adenocarinoma cells and endothelial cells. Some studies disclosed that the integrinbinding domains located in FN were different from the DPP IV-binding sites, indicating that they may operate in a different manner $(32,33)$. DPP IV might not be an indispensable receptor for FN in mediating adhesion between lung adenocarcinoma cells and endothelial cells.

As stated above, our results offered a scheme by which AAT facilitates lung adenocarcinoma metastasis. As shown in Fig. 5, lung adenocarcinoma cells may express high levels of AAT. As AAT is the most abundant proteinase inhibitor within the lung, it may inhibit the proteolytic activity of proteinases targeting at FN, and recruit more FN overexpressed on the surface of cancer cells. This situation may further increase the adhesion of adenocarcinoma cells to endothelial cells through $\mathrm{FN}$ interacting with integrins. Binding of FN to integrins may not only serve as a way for cell adhesion, but also generate the traction needed for cell migration.

Noteworthy, it has been shown that AAT could have an antiapoptotic role in alveolar cells (34). Under certain conditions, inhibition of apoptosis in lung tissue by AAT may become a pathological mechanism that leads to lung cancer development and metastasis. On the other hand, AAT could be produced by various tumor cells. Therefore, one can speculate a link between tumor cell propensity to produce and secrete AAT and tumor progression or metastasis. Additionally, AAT may mediate immune tolerance (35), and might help cancer cells escape immune surveillance to metastasis. FN upregulation has been found in several types of malignant tumors and its high expression positively correlates with metastasis $(36,37)$. AAT may modulate the expression of FN through signaling pathways. This may represent crosstalk that is of prognostic relevance in lung cancer. The association between FN and AAT needs further research.

In conclusion, our study explored the mechanisms by which AAT promotes lung adenocarcinoma metastasis and identified the effect of FN during the processes. Our findings offer useful information for an understanding of the mechanisms of lung adenocarcinoma metastasis.

\section{Acknowledgements}

We would like to thank Dr Kui Meng (Department of Pathology, Nanjing Drum Tower Hospital, The Affiliated Hospital of Nanjing University Medical School, Nanjing, China) for his technical support in the evaluation of the pathological samples. This work was supported by the grants from the Natural Science Foundation of Jiangsu Province (no. BK20130089), the National Natural Science Foundation of China (no. 81501972), and the Nanjing Municipal Health and Family Planning Commission (no. YKK15063).

\section{References}

1. Travis WD: Pathology of lung cancer. Clin Chest Med 23: 65-81, viii, 2002

2. Hanahan D and Weinberg RA: The hallmarks of cancer. Cell 100: 57-70, 2000

3. Comunale MA, Rodemich-Betesh L, Hafner J, Wang M, Norton P, Di Bisceglie AM, Block T and Mehta A: Linkage specific fucosylation of alpha-1-antitrypsin in liver cirrhosis and cancer patients: Implications for a biomarker of hepatocellular carcinoma. PLoS One 5: e12419, 2010.

4. El-Akawi ZJ, Abu-Awad AM, Sharara AM and Khader Y: The importance of alpha-1 antitrypsin (alpha1-AT) and neopterin serum levels in the evaluation of non-small cell lung and prostate cancer patients. Neuro Endocrinol Lett 31: 113-116, 2010. 
5. Zelvyte I, Wallmark A, Piitulainen E, Westin U and Janciauskiene S: Increased plasma levels of serine proteinase inhibitors in lung cancer patients. Anticancer Res 24: 241-247, 2004.

6. Ioachim E, Charchanti A, Briasoulis E, Karavasilis V, Tsanou H, Arvanitis DL, Agnantis NJ and Pavlidis N: Immunohistochemical expression of extracellular matrix components tenascin, fibronectin, collagen type IV and laminin in breast cancer: Their prognostic value and role in tumour invasion and progression. Eur J Cancer 38: 2362-2370, 2002.

7. David L, Nesland JM, Holm R and Sobrinho-Simões M: Expression of laminin, collagen IV, fibronectin, and type IV collagenase in gastric carcinoma. An immunohistochemical study of 87 patients. Cancer 73: 518-527, 1994.

8. Pankov R and Yamada KM: Fibronectin at a glance. J Cell Sci 115: 3861-3863, 2002.

9. Williams CM, Engler AJ, Slone RD, Galante LL and Schwarzbauer JE: Fibronectin expression modulates mammary epithelial cell proliferation during acinar differentiation. Cancer Res 68: 3185-3192, 2008

10. Han S, Khuri FR and Roman J: Fibronectin stimulates non-small cell lung carcinoma cell growth through activation of Akt/ mammalian target of rapamycin/S6 kinase and inactivation of LKB1/AMP-activated protein kinase signal pathways. Cancer Res 66: 315-323, 2006.

11. Alizadeh AM, Shiri S and Farsinejad S: Metastasis review: From bench to bedside. Tumour Biol 35: 8483-8523, 2014.

12. Burgett ME, Lathia JD, Roth P, Nowacki AS, Galileo DS, Pugacheva E, Huang P, Vasanji A, Li M, Byzova T, et al: Direct contact with perivascular tumor cells enhances integrin $\alpha v \beta 3$ signaling and migration of endothelial cells. Oncotarget 7: 43852-43867, 2016.

13. Schwarzbauer JE and DeSimone DW: Fibronectins, their fibrillogenesis, and in vivo functions. Cold Spring Harb Perspect Biol 3: a005041, 2011.

14. Hartel-Schenk S, Gossrau R and Reutter W: Comparative immunohistochemistry and histochemistry of dipeptidyl peptidase IV in rat organs during development. Histochem J 22: 567-578, 1990.

15. Johnson RC, Zhu D, Augustin-Voss HG and Pauli BU: Lung endothelial dipeptidyl peptidase IV is an adhesion molecule for lung-metastatic rat breast and prostate carcinoma cells. J Cell Biol 121: 1423-1432, 1993.

16. Piazza GA, Callanan HM, Mowery J and Hixson DC: Evidence for a role of dipeptidyl peptidase IV in fibronectin-mediated interactions of hepatocytes with extracellular matrix. Biochem J 262: 327-334, 1989.

17. Cheng HC, Abdel-Ghany M, Elble RC and Pauli BU: Lung endothelial dipeptidyl peptidase IV promotes adhesion and metastasis of rat breast cancer cells via tumor cell surface-associated fibronectin. J Biol Chem 273: 24207-24215, 1998.

18. Livak KJ and Schmittgen TD: Analysis of relative gene expression data using real-time quantitative PCR and the 2(-Delta Delta C(T)) method. Methods 25: 402-408, 2001.

19. Sun Z and Yang P: Role of imbalance between neutrophil elastase and alpha 1-antitrypsin in cancer development and progression. Lancet Oncol 5: 182-190, 2004.

20. Chen XL, Zhou L, Yang J, Shen FK, Zhao SP and Wang YL: Hepatocellular carcinoma-associated protein markers investigated by MALDI-TOF MS. Mol Med Rep 3: 589-596, 2010.

21. Li Y, Krowka MJ, Qi Y, Katzmann JA, Song Y, Li Y, Mandrekar SJ and Yang P: Alpha1-antitrypsin deficiency carriers, serum alpha1-antitrypsin concentration, and non-small cell lung cancer survival. J Thorac Oncol 6: 291-295, 2011.
22. Jia D, Yan M, Wang X, Hao X, Liang L, Liu L, Kong H, He X, $\mathrm{Li} \mathrm{J}$ and Yao M: Development of a highly metastatic model that reveals a crucial role of fibronectin in lung cancer cell migration and invasion. BMC Cancer 10: 364, 2010.

23. Cao Y, Liu X, Lu W, Chen Y, Wu X, Li M, Wang XA, Zhang F, Jiang L, Zhang Y, et al: Fibronectin promotes cell proliferation and invasion through mTOR signaling pathway activation in gallbladder cancer. Cancer Lett 360: 141-150, 2015.

24. Digiacomo G, Tusa I, Bacci M, Cipolleschi MG, Dello Sbarba P and Rovida E: Fibronectin induces macrophage migration through a SFK-FAK/CSF-1R pathway. Cell Adh Migr 2: 1-11, 2016.

25. Vakonakis I and Campbell ID: Extracellular matrix: From atomic resolution to ultrastructure. Curr Opin Cell Biol 19: 578-583, 2007.

26. Yousif NG: Fibronectin promotes migration and invasion of ovarian cancer cells through up-regulation of FAK-PI3K/Akt pathway. Cell Biol Int 38: 85-91, 2014.

27. Mitra AK, Sawada K, Tiwari P, Mui K, Gwin K and Lengyel E: Ligand-independent activation of c-Met by fibronectin and $\alpha(5)$ $\beta(1)$-integrin regulates ovarian cancer invasion and metastasis. Oncogene 30: 1566-1576, 2011.

28. Maity G, Choudhury PR, Sen T, Ganguly KK, Sil H and Chatterjee A: Culture of human breast cancer cell line (MDAMB-231) on fibronectin-coated surface induces pro-matrix metalloproteinase-9 expression and activity. Tumour Biol 32: 129-138, 2011.

29. Subbaram S and Dipersio CM: Integrin $\alpha 3 \beta 1$ as a breast cancer target. Expert Opin Ther Targets 15: 1197-1210, 2011.

30. Han S, Sidell N and Roman J: Fibronectin stimulates human lung carcinoma cell proliferation by suppressing p 21 gene expression via signals involving Erk and Rho kinase. Cancer Lett 219: 71-81, 2005.

31. Knowles LM, Gurski LA, Engel C, Gnarra JR, Maranchie JK and Pilch J: Integrin $\alpha v \beta 3$ and fibronectin upregulate Slug in cancer cells to promote clot invasion and metastasis. Cancer Res 73: 6175-6184, 2013

32. Mohri H: Interaction of fibronectin with integrin receptors: Evidence by use of synthetic peptides. Peptides 18: 899-907, 1997.

33. Cheng HC, Abdel-Ghany M and Pauli BU: A novel consensus motif in fibronectin mediates dipeptidyl peptidase IV adhesion and metastasis. J Biol Chem 278: 24600-24607, 2003

34. Petrache I, Fijalkowska I, Zhen L, Medler TR, Brown E, Cruz P, Choe KH, Taraseviciene-Stewart L, Scerbavicius R, Shapiro L, et al: A novel antiapoptotic role for alpha1-antitrypsin in the prevention of pulmonary emphysema. Am J Respir Crit Care Med 173: 1222-1228, 2006.

35. Ozeri E, Mizrahi M, Shahaf G and Lewis EC: $\alpha-1$ antitrypsin promotes semimature, IL-10-producing and readily migrating tolerogenic dendritic cells. J Immunol 189: 146-153, 2012.

36. Malik G, Knowles LM, Dhir R, Xu S, Yang S, Ruoslahti E and Pilch J: Plasma fibronectin promotes lung metastasis by contributions to fibrin clots and tumor cell invasion. Cancer Res 70: 4327-4334, 2010

37. Lal A, Lash AE, Altschul SF, Velculescu V, Zhang L, McLendon RE, Marra MA, Prange C, Morin PJ, Polyak K, et al: A public database for gene expression in human cancers. Cancer Res 59: 5403-5407, 1999. 